\title{
Genetic Variation for Grain Yield and Related Traits in Temperate Red Rice (Oryza sativa L.) Ecotypes
}

\author{
Gulzar S. SANGHERA*, Subhash C. KASHYAP, Ghulam A. PARRAY \\ SKUAST-Kashmir, Mountain Research Centre for Field Crops, Khudwani, Anantnag, 192102, \\ $J$ and K, India; g_singh72@rediffmail.com (*corresponding author)
}

\begin{abstract}
The present study was carried out in Kashmir (India) to assess the genetic variability for grain yield and component traits among 14 red rice ecotypes from temperate region (locally known as Zag for its coloured kernels) and correlation and path coefficients were also studied for fifteen agro-morphological characters. Genotypic and phenotypic coefficients of variation were high for grain yield, secondary branches per panicle and panicle weight; moderate for grain number per panicle, grain length:breadth (L:B) ratio and panicle density. High heritability accompanied by high to moderate genetic advance for panicle density, days to $50 \%$ flowering, plant height, grain number indicated the predominance of additive gene action for the expression of these characters. Grain yield was found to be positively and significantly correlated with number of tiller per plant, panicle density $\mathrm{m}^{-2}$ and number of grain per panicle at both genotypic and phenotypic levels indicating the importance of these characters for yield improvement in this material. The results of genotypic path analysis revealed that panicle density had the highest positive direct effect followed by plant height and days to flower. The overall results indicated that selection favouring higher panicle density, test weight and panicle weight and medium plant height with a reasonable balance for moderate grain number would help to achieve higher grain yield in this population of red rice ecotypes.
\end{abstract}

Keywords: correlation, genetic advance, heritability, path coefficients, red rice ecotypes, temperate conditions

\section{Introduction}

Agriculture in developing countries plays a central role in securing livelihoods by providing food, clothes and medicine. In this context, rice (Oryza sativa L.) is the most important cereal crop of the world which is consumed as a staple food by over one-half of the world's population with approximately $95 \%$ of production in Asia (Bhattacharjee et al., 2002). In the traditional growing areas of Asia, rice of various colors - red, purple, black, brown, yellow, and green - have been known and grown, but for the present-day generation, rice connotes pearly white grain. Their name refer to the kernel colour (black, red or purple) which is formed by deposits of anthocyanins in different layers of the pericarp, seed coat and aleurone (Chaudhary, 2003). Colored rice have been preferred in the past for their special features such as medicinal value and exclusive taste. Flavored, black rice were the favorites of the royals of China, while red rice were preferred by people in many parts of India, Sri Lanka, and Bhutan (Oka, 1988). Such rice includes many forms of genetic resources in relation to food and agriculture including wild and cultivated varieties and landraces. Landraces are products selected by farmers for their various forms of production benefits. Landrace is a variety bred and cultivated by farmers and adapted to local conditions. These have specific traits that satisfy specific needs but all components of the population are adapted to local climatic conditions, cultural practices, and disease and pests. Unlike distinct, uniform and stabilized modern varieties with wider adaptation, landraces are considered to have a narrow range of adaptation as they were developed through natural and human selection over time. Rice with a red bran layer is called red rice, though the color is confined to the bran layer, a tinge of red remains even after a high degree of milling. The color of the bran ranges from light to dark red. The bran layer contains polyphenols and anthocyanin, and possesses antioxidant properties. The zinc and iron content of red rices is 2-3 times higher than that of white rices (Rood, 2000). With the sharp increase in lifestyle-related health issues and diseases - such as diabetes, cancer, and heart problems - scientists are looking at quality traits other than carbohydrates, protein, and fat in foodstuffs (Zhang et al., 2005). Red rices have been found to have greater antioxidant property than black and white rices. With this rediscovery of their nutritive and medicinal value, red rices have begun to regain their old position and prestige. There is a revival of red rices in Japan, and new cultivars are being released that are suitable for preparations such as red sake, noodles, and rice cakes (Itani and Ogawa, 2004). Red rice (locally known as Zag because of its coloured kernels) is one such land race of rice of Kashmir possessing the characteristic coloured pericarp and enhanced nutrient quality making it a prized variety in Kashmir valley. Therefore, the present investigation was undertaken for genetic evaluation of 14 morphologically distinct red rice ecotypes collected from temperate region 
of Jammu and Kashmir, India. It is well known that breeding for yield component for increasing grain yield would be most effective, if the components involved are highly heritable and genetically independent or positively correlated with grain yield. However, it is very difficult to judge whether observed variability is highly heritable or not. So, knowledge of heritability is essential for selection based improvement as it indicates the extent of transmissibility of a character into future generations (Sabesan et al., 2009). Since, grain yield and quality are complex characters and are associated with number of component characters, which themselves are interrelated. Such dependence often affects their relationship with yield, thereby making correlation ineffective. Further, there is a need for path analysis that permits the partitioning of the correlation coefficient into its components, one component being the path coefficient that measures the direct effect of a predictor variable upon its response variable; the second component being the indirect effect of a predictor variable on the response variable through another predictor variable. Therefore, the present study reports the extent of genetic variability, heritability estimates and degree of association of grain yield and yield related traits in red rice ecotypes grown under temperate conditions.

\section{Materials and methods}

The experimental material consisted of fourteen diverse cold tolerant local red rice ecotypes designated as 'ZAG1', 'ZAG2', 'ZAG3', 'ZAG4', 'ZAG5', 'ZAG6', 'ZAG7', 'ZAG8', 'ZAG9', 'ZAG10', 'ZAG11', 'ZAG12', 'ZAG13' and 'ZAG14' (Fig. 1) (Z stand for Zag a local name for red rice). The experiment was evaluated during wet season 2011 and 2012 at SKUAST-Kashmir, Mountain Research Centre for Field Crops, Khudwani, Anantnag (India) at an elevation of $1560 \mathrm{~m}$ amsl (above mean sea level) in randomized complete block design with three replications. Thirty days old seedlings were transplanted with an inter-row and intra-row spacing of $20 \mathrm{~cm}$ and 15 $\mathrm{cm}$, respectively. Recommended package of practices were followed for raising ideal crop stand. Observations were recorded on yield and its contributing characters. Five representative hills for each genotypes in each replications were randomly selected to record the observations for twelve quantitative traits viz., plant height $(\mathrm{cm})$, number of tillers per plant, panicle length $(\mathrm{cm})$, no. of primary branches per panicle, number of secondary branches per panicle, no. of grain per panicle, panicle weight (g), 100 grain weight (g) while data for days to $50 \%$ flowering, days to maturity and yield were recorded on per plot basis and panicle density per square meter. The mean data were put to statistical analysis for variability parameters (PCV, GCV, heritability and genetic advance) and correlation and path analysis as per methods given by Johnson et al. (1955) and Dewey and Lu (1959), respectively.

\section{Results and discussion}

A wide range of variation was observed among all fourteen rice (Oryza sativa L.) ecotypes for fifteen quantitative characters as well as pericarp color which ranges from light cream to dark red (Fig. 1). Analysis of variance showed significant difference among ecotypes for all the traits studied and revealed a wide range of variability (Tab. 1). Days to $50 \%$ flowering shows significant differences among all genotypes ranged from 80 to 97 days after sowing and for days to maturity which ranged between 124 to 129 days. Ecotype 'ZAG 6' found to be the early in maturity (121 days) while genotype 'ZAG2' was late in maturity (138 days). Plant height showed significant difference among all the genotypes. It ranged from $89.66 \mathrm{~cm}$ ('ZAG13') to $141.4 \mathrm{~cm}$ ('ZAG2').The number of tillers per plant ranges

Tab. 1. Parameters of variability studied for fifteen agro-morphological characters in red rice ecotypes from temperate region of Kashmir, India

\begin{tabular}{|c|c|c|c|c|c|}
\hline Characters & Range & $\mathrm{PCV}$ & $\mathrm{GCV}$ & Heritability (bs) \% & Genetic Advance (\%) \\
\hline Days to $50 \%$ flowering & $80-97$ & 6.10 & 5.88 & 92.82 & 31.68 \\
\hline Days to maturity & $121-138$ & 3.40 & 3.30 & 94.17 & 36.60 \\
\hline Plant height $(\mathrm{cm})$ & $89.66-141.4$ & 14.55 & 14.53 & 79.71 & 29.89 \\
\hline Number of tillers per plant & $9.33-15.26$ & 17.99 & 15.19 & 71.31 & 26.43 \\
\hline Panicle density $\left(\mathrm{m}^{-2}\right)$ & $230-450$ & 26.08 & 24.86 & 70.84 & 38.82 \\
\hline Panicle length $(\mathrm{cm})$ & $15.4-23.4$ & 10.02 & 8.74 & 56.20 & 15.73 \\
\hline No. of primary branches per panicle & $7.2-9.8$ & 13.04 & 5.39 & 57.10 & 24.59 \\
\hline No. of secondary branches per panicle & $7-20.8$ & 36.25 & 35.24 & 54.50 & 26.57 \\
\hline No. of grain per panicle & $79.4-157.8$ & 20.38 & 20.36 & 69.78 & 21.90 \\
\hline Panicle weight $(\mathrm{g})$ & $6.63-17.81$ & 28.15 & 26.85 & 80.97 & 32.75 \\
\hline 100 grain weight $(\mathrm{g})$ & $2.29-3.77$ & 14.00 & 13.21 & 89.02 & 25.68 \\
\hline Grain length (mm) & $3.07-4.01$ & 12.11 & 12.07 & 79.41 & 34.80 \\
\hline Grain breadth (mm) & $1.05-2.01$ & 29.26 & 29.17 & 79.36 & 39.90 \\
\hline $\mathrm{L}: \mathrm{B}$ ratio & $1.53-3.71$ & 20.18 & 20.17 & 89.89 & 31.53 \\
\hline Grain yield (q/ha) & $46-67.5$ & 30.91 & 30.87 & 58.72 & 23.51 \\
\hline
\end{tabular}


402

between 8.80 and 15.26 ('ZAG13'). The number of panicles per $\mathrm{m}^{-2}$ was significantly affected by genotypes and varied from 230 in 'ZAG2' to 450 in genotype 'ZAG13'. Number of grains per panicle is one of the most important components of yield. It differs significantly among all the tested genotypes. The highest number of grains per panicle (157.8) was recorded in ecotype 'ZAG2' followed by 'ZAG12' (136) and 'ZAG5' (120). The highest grain yield $(67.5 \mathrm{q} / \mathrm{ha})$ was observed in 'ZAG5' while the lowest grain yield ( $46 \mathrm{q} / \mathrm{ha}$ ) was recorded in genotype 'ZAG10'. The genetic variability for panicle length and other panicle traits in different red rice ecotypes from temperate region of Kashmir is shown in Fig. 2. The morphological features of all the ecotypes are presented in Tab. 2.
Phenotypic variance was higher than the genotypic variances for all the characters thus indicated the influences of environmental factor on these. The extent of the environmental influence on any character is indicated by the magnitude of the differences between the genotypic and phenotypic coefficients of variation. Sinha et al. (2004) also observed similar results and concluded that large differences reflect high environmental influence, while small differences reveal high genetic influence. Moderate to low genotypic and phenotypic coefficients of variation obtained for days to $50 \%$ flowering, days to maturity, plant height and panicle length these findings are further supported by Iftekharuddeula et al. (2001). The small differences observed between genotypic and phenotypic coef-

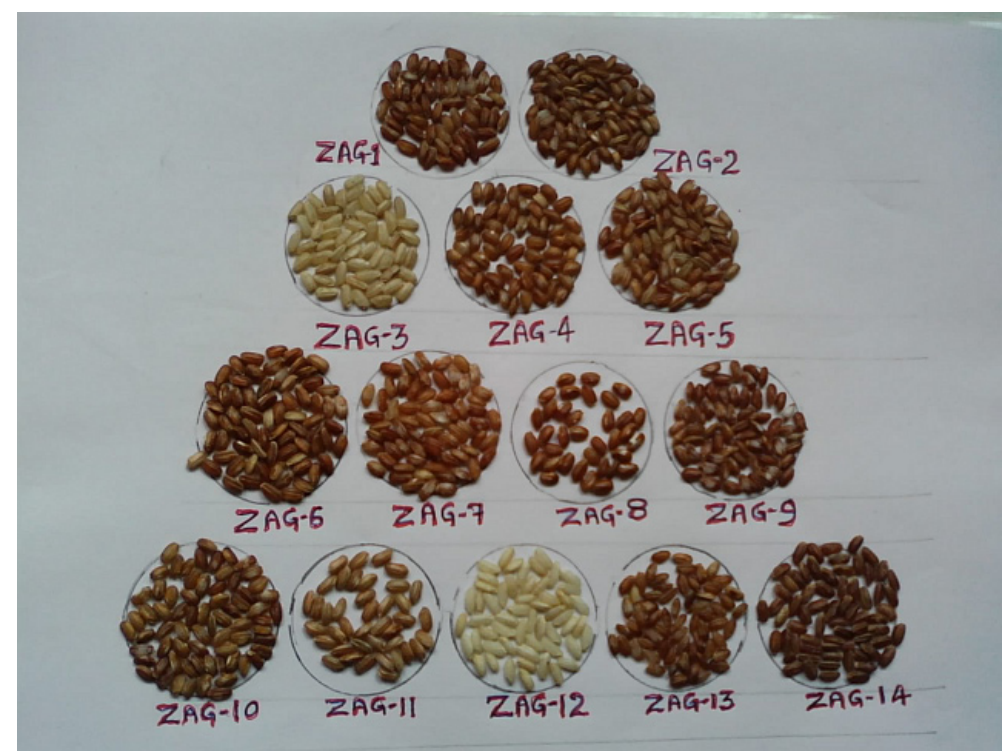

Fig. 1. Variability in pericarp colour intensity among 14 red rice ecotypes from temperate region of Kashmir, India

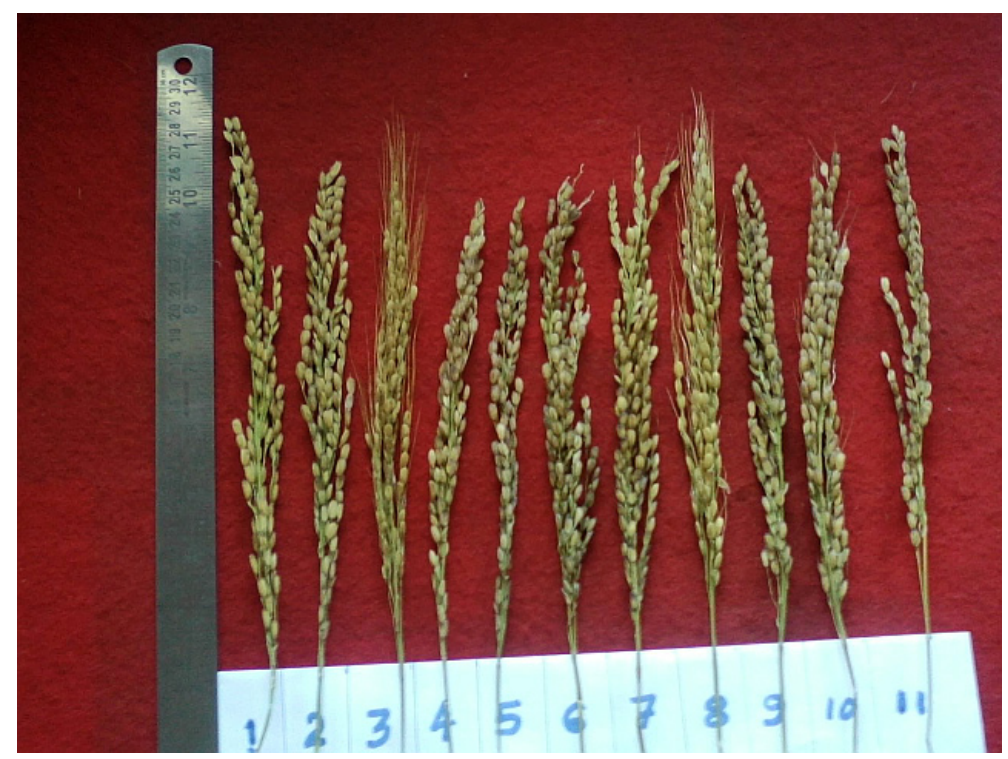

Fig. 2. Genetic variability for panicle length and other panicle traits in different red rice ecotypes from temperate region of Kashmir, India 
Tab. 2. Morphological characters studied for 14 red rice ecotypes from temperate region of Kashmir, India

\begin{tabular}{|c|c|c|c|c|c|c|c|c|c|c|}
\hline $\begin{array}{l}\text { S. } \\
\text { no }\end{array}$ & Designation & $\begin{array}{c}\text { Flag leaf } \\
\text { angle }\end{array}$ & $\begin{array}{l}\text { Auricle } \\
\text { colour }\end{array}$ & $\begin{array}{c}\text { Ligule } \\
\text { shape }\end{array}$ & $\begin{array}{l}\text { Plant } \\
\text { type }\end{array}$ & $\begin{array}{l}\text { Panicle } \\
\text { exersion }\end{array}$ & $\begin{array}{c}\text { Panicle } \\
\text { type }\end{array}$ & Awn & $\begin{array}{c}\text { Awn } \\
\text { colour }\end{array}$ & Pubescence \\
\hline 1 & 'ZAG1' & horizontal & green & Clift & compact & full & compact & awnless & purple & Absent \\
\hline 2 & 'ZAG2' & drooping & green & Clift & compact & full & compact & awnless & purple & Absent \\
\hline 3 & 'ZAG3' & erect & green & normal & compact & full & compact & awned & purple & Present \\
\hline 4 & 'ZAG4' & drooping & green & Clift & compact & good & compact & Rudimentary awned & purple & Present \\
\hline 5 & 'ZAG5' & horizontal & green & normal & open & good & compact & long awn & purple & Present \\
\hline 6 & 'ZAG6' & horizontal & green & Clift & open & Just exerted & compact & awned & purple & Present \\
\hline 7 & 'ZAG7' & horizontal & green & normal & open & exerted & compact & awned & purple & Present \\
\hline 8 & 'ZAG8' & horizontal & green & Clift & open & Well exerted & compact & long awn & white & Present \\
\hline 9 & 'ZAG9' & horizontal & green & Clift & open & Well exerted & open & long awn & white & Absent \\
\hline 10 & 'ZAG10’ & horizontal & green & Clift & compact & Well exerted & compact & long awn & white & Absent \\
\hline 11 & 'ZAG11' & horizontal & green & Clift & compact & Well exerted & compact & awnless & white & Present \\
\hline 12 & 'ZAG12' & erect & green & Clift & compact & full & compact & awnless & white & Absent \\
\hline 13 & 'ZAG13' & erect & green & Clift & compact & full & compact & awned & white & Present \\
\hline 14 & 'ZAG14' & horizontal & purple & Clift & compact & full & compact & awned & purple & Present \\
\hline
\end{tabular}

ficients of variation on days to heading, days to maturity, plant height and panicle length indicate the presence of sufficient genetic variability for the traits which may facilitate selection (Yadav, 2000) for the improvement of these traits especially under temperate environment. The high to moderate differences between genotypic and phenotypic coefficients of variation observed in number of panicles density per square meter, number of tillers per plant, panicle weight, number of grains per panicle, 100 grain weight and grain yield indicate high influence of the environment on the traits which are in agreement with results have already been reported (Bhandarkar et al., 2002; Kuldeep et al., 2004; Sabesan et al., 2009; Jayasudha and Sharma, 2010).

Heritability in broad sense is the relative magnitude of genotypic and phenotypic variances for the traits and plays a predictive role in selection procedures. This gives an idea of the total variation ascribable to genotypic effects, which are exploitable portion of variation. The heritability estimates among the traits studied ranged from 59.72 to $99.14 \%$ (Tab. 1). High to medium broad sense heritability estimates observed for days to heading, days to maturity, plant height, number of grains per panicle, panicle weight, panicle density per square meter. These results are in consonance with the findings (Babu et al., 2011; Prajapati et al., 2011; Idris et al., 2012). It suggests high component of heritable portion of variation plays a vital role in deciding the suitability and strategy for selection of a particular character. The low broad sense heritability observed for the number of primary branches per panicle, secondary branches per panicle, panicle length and grain yield indicates the influence of the environment on manifestation of these traits. The low heritability recorded for these traits indicates that direct selection for these traits will be ineffective. Since high heritability does not always indicate high genetic gain, heritability with genetic advance considered together should be used in predicting the ultimate effect for selecting superior genotypes for developing a variety. High heritability coupled with genetic advance was recorded for traits days to $50 \%$ flowering and maturity, plant height, panicle density, panicle weight, grain length, grain width and L: B ratio while moderate genetic advance was exhibited by primary branches per panicle, secondary branches per panicle, grain per panicle and 100 grain weight. Bhardwaj et al. (2007) have also reported high to moderate heritability and genetic advance for these traits that needs to be exploited by breeders for selecting superior genotypes.

Further, knowledge on interrelationship of grain yield with other characters is of paramount importance to the breeder for making improvement in complex quantitative character like grain yield for which direct selection is not much effective. In present study, association analysis revealed that the genotypic correlation coefficients were higher in magnitude than phenotypic correlation coefficients which are due to the masking effect of environment in genetic association between the characters. Grain yield was positively and significantly associated with panicle length $(G=0.831, P=0.830)$, number of grains per panicle $(\mathrm{G}=0.356, \mathrm{P}=0.352)$, number of tiller per plant $(\mathrm{G}=0.520$, $\mathrm{P}=0.454)$, panicle density $(\mathrm{G}=0.340, \mathrm{P}=0.317)$ at both genotypic and phenotypic levels (Tab. 3) indicating the importance of these characters for yield improvement. Therefore, direct selection of these characteristics would be undertaken for improving grain yield in this population of red rice ecotypes under temperate conditions. Significant positive relationship of these traits with grain yield have been documented in rice by earlier researchers (Siva Kumar and Kannan Bapu, 2005; Eradasappa et al., 2007; Sanghera and Kashyap, 2012). It is also desirable to select genotypes with more productive tillers per plant with more panicle length having more number of grains because of their positive and significant associations with each other as evidenced in this study. The results pertaining to path 
Tab. 3. Phenotypic (P) and genotypic $(\mathrm{G})$ correlation among fifteen agro-morphological characters under study in red rice ecotypes from temperate region of Kashmir, India

\begin{tabular}{|c|c|c|c|c|c|c|c|c|c|c|c|c|c|c|c|}
\hline Characters & & DF & $\mathrm{DM}$ & $\mathrm{PH}$ & NT & $\mathrm{PD}$ & $\mathrm{PB}$ & SB & $\mathrm{NG}$ & $\mathrm{PL}$ & $\mathrm{PW}$ & GW & $\mathrm{GL}$ & $\mathrm{GB}$ & $\mathrm{L}: \mathrm{B}$ \\
\hline \multirow{2}{*}{$\mathrm{DM}$} & $\mathrm{P}$ & $0.140^{\mathrm{NS}}$ & & & & & & & & & & & & & \\
\hline & G & $0.156^{\mathrm{NS}}$ & & & & & & & & & & & & & \\
\hline \multirow{2}{*}{$\mathrm{PH}$} & $\mathrm{P}$ & $0.251^{\mathrm{NS}}$ & $0.258^{\mathrm{NS}}$ & & & & & & & & & & & & \\
\hline & G & $0.260^{\mathrm{NS}}$ & $0.268^{\mathrm{NS}}$ & & & & & & & & & & & & \\
\hline \multirow{2}{*}{ NT } & $\mathrm{P}$ & $0.180^{\mathrm{NS}}$ & $0.182^{\mathrm{NS}}$ & $-0.463 "$ & & & & & & & & & & & \\
\hline & G & $0.214^{\mathrm{NS}}$ & $0.194^{\mathrm{NS}}$ & $-0.552^{*}$ & & & & & & & & & & & \\
\hline \multirow{2}{*}{ PD } & $\mathrm{P}$ & $0.159^{\mathrm{NS}}$ & $-0.010^{\mathrm{NS}}$ & -0.684 & $0.697^{*}$ & & & & & & & & & & \\
\hline & G & $0.160^{\mathrm{NS}}$ & $-0.031^{\mathrm{NS}}$ & $-0.718^{*}$ & $0.898^{* \prime}$ & & & & & & & & & & \\
\hline \multirow{2}{*}{$\mathrm{PB}$} & $\mathrm{P}$ & $-0.029^{\mathrm{NS}}$ & $0.172^{\mathrm{NS}}$ & $0.655^{\prime \prime}$ & -0.704 & $-0.660^{\prime \prime}$ & & & & & & & & & \\
\hline & G & $-0.132^{\mathrm{NS}}$ & $0.235^{\mathrm{NS}}$ & $0.750^{\prime \prime}$ & $-0.883^{\prime \prime}$ & $-0.829^{\prime \prime}$ & & & & & & & & & \\
\hline \multirow{2}{*}{ SB } & $\mathrm{P}$ & $0.061^{\mathrm{NS}}$ & $-0.036^{\mathrm{NS}}$ & $-0.068^{\mathrm{NS}}$ & $-0.129^{\mathrm{NS}}$ & $0.076^{\mathrm{NS}}$ & $0.110^{\mathrm{NS}}$ & & & & & & & & \\
\hline & G & $0.007^{\mathrm{NS}}$ & $-0.101^{\mathrm{NS}}$ & $-0.115^{\mathrm{NS}}$ & $-0.181^{\mathrm{NS}}$ & $-0.042^{\mathrm{NS}}$ & $0.166^{\mathrm{NS}}$ & & & & & & & & \\
\hline \multirow{2}{*}{ NG } & $\mathrm{P}$ & $0.708^{* \prime}$ & $0.262^{\mathrm{NS}}$ & $0.420^{\prime \prime}$ & $-0.009^{\mathrm{NS}}$ & $0.022^{\mathrm{NS}}$ & $0.134^{\mathrm{NS}}$ & $0.000^{\mathrm{NS}}$ & & & & & & & \\
\hline & G & $0.779^{\prime \prime}$ & $0.265^{\mathrm{NS}}$ & $0.435^{\prime \prime}$ & $-0.017^{\mathrm{NS}}$ & $0.044^{\mathrm{NS}}$ & $0.176^{\mathrm{NS}}$ & $-0.137^{\mathrm{NS}}$ & & & & & & & \\
\hline \multirow{2}{*}{ PL } & $\mathrm{P}$ & $0.194^{\mathrm{NS}}$ & $0.427^{*}$ & $0.415^{\prime \prime}$ & $-0.351^{\circ}$ & $-0.370^{\circ}$ & 0.556 & 0.336 & $0.352^{\circ}$ & & & & & & \\
\hline & G & $0.202^{\mathrm{NS}}$ & 0.446 & 0.416 & $-0.418^{* \prime}$ & -0.384 & $0.640^{*}$ & $0.803^{*}$ & $0.365^{\circ}$ & & & & & & \\
\hline \multirow{2}{*}{ PW } & $\mathrm{P}$ & $0.048^{\mathrm{NS}}$ & $0.334^{\circ}$ & $0.260^{\mathrm{NS}}$ & $-0.302^{\mathrm{NS}}$ & $-0.444^{*}$ & $0.380^{\circ}$ & $0.260^{\mathrm{NS}}$ & $-0.101^{\mathrm{NS}}$ & 0.572 & & & & & \\
\hline & G & $0.070^{\mathrm{NS}}$ & 0.356 & $0.277^{\mathrm{NS}}$ & $-0.424^{\prime \prime}$ & $-0.445^{\prime \prime}$ & $0.547^{\circ}$ & $0.493^{*}$ & $-0.114^{\mathrm{NS}}$ & 0.596 & & & & & \\
\hline \multirow{2}{*}{ GB } & $\mathrm{P}$ & $-0.140^{\mathrm{NS}}$ & $-0.339^{\circ}$ & $0.159^{\mathrm{NS}}$ & $-0.491^{*}$ & -0.326 & $0.372^{\circ}$ & $-0.085^{\mathrm{NS}}$ & $0.024^{\mathrm{NS}}$ & $-0.083^{\mathrm{NS}}$ & $-0.106^{\mathrm{NS}}$ & & & & \\
\hline & G & $-0.153^{\mathrm{NS}}$ & $-0.368^{\circ}$ & $0.172^{\mathrm{NS}}$ & $-0.573^{\prime \prime}$ & $-0.414^{\prime \prime}$ & $0.437^{\prime \prime}$ & $-0.568^{\prime \prime}$ & $0.028^{\mathrm{NS}}$ & $-0.090^{\mathrm{NS}}$ & $-0.129^{\mathrm{NS}}$ & & & & \\
\hline \multirow{2}{*}{ GL } & $\mathrm{P}$ & $-0.188^{\mathrm{NS}}$ & $-0.305^{\circ}$ & $-0.059^{\mathrm{NS}}$ & $-0.302^{\mathrm{NS}}$ & $-0.198^{\mathrm{NS}}$ & $0.394^{\prime \prime}$ & $0.198^{\mathrm{NS}}$ & $-0.156^{\mathrm{NS}}$ & $0.172^{\mathrm{NS}}$ & $0.089^{\mathrm{NS}}$ & $0.577^{*}$ & & & \\
\hline & G & $-0.189^{\mathrm{NS}}$ & $-0.307^{\circ}$ & $-0.060^{\mathrm{NS}}$ & $-0.351^{*}$ & $-0.206^{\mathrm{NS}}$ & $0.448^{\prime \prime}$ & $0.505^{*}$ & $-0.164^{\mathrm{NS}}$ & $0.172^{\mathrm{NS}}$ & $0.099^{\mathrm{NS}}$ & $0.607^{*}$ & & & \\
\hline \multirow{2}{*}{ GW } & $\mathrm{P}$ & $-0.589^{\prime \prime}$ & $-0.308^{*}$ & $-0.227^{\mathrm{NS}}$ & $-0.281^{\mathrm{NS}}$ & $-0.311^{\circ}$ & $0.021^{\mathrm{NS}}$ & $0.056^{\mathrm{NS}}$ & $-0.553^{*}$ & $-0.097^{\mathrm{NS}}$ & $0.068^{\mathrm{NS}}$ & $0.238^{\mathrm{NS}}$ & $0.088^{\mathrm{NS}}$ & & \\
\hline & G & $-0.610^{\prime \prime}$ & $-0.325^{\circ}$ & $-0.228^{\mathrm{NS}}$ & $-0.350^{\circ}$ & $-0.328^{\circ}$ & $0.031^{\mathrm{NS}}$ & $0.147^{\mathrm{NS}}$ & $-0.571^{*}$ & $-0.097^{\mathrm{NS}}$ & $0.074^{\mathrm{NS}}$ & $0.264^{\mathrm{NS}}$ & $0.090^{\mathrm{NS}}$ & & \\
\hline \multirow{2}{*}{$\mathrm{L}: \mathrm{B}$} & $\mathrm{P}$ & $0.387^{\circ}$ & $0.106^{\mathrm{NS}}$ & $0.149^{\mathrm{NS}}$ & $0.064^{\mathrm{NS}}$ & $0.178^{\mathrm{NS}}$ & $0.194^{\mathrm{NS}}$ & $-0.005^{\mathrm{NS}}$ & $0.384^{\circ}$ & $0.111^{\mathrm{NS}}$ & $0.044^{\mathrm{NS}}$ & $0.177^{\mathrm{NS}}$ & $0.446^{\circ}$ & -0.816 & \\
\hline & G & $0.401^{*}$ & $0.111^{\mathrm{NS}}$ & $0.149^{\mathrm{NS}}$ & $0.075^{\mathrm{NS}}$ & $0.185^{\mathrm{NS}}$ & $0.216^{\mathrm{NS}}$ & $-0.005^{\mathrm{NS}}$ & $0.394^{\prime \prime}$ & $0.111^{\mathrm{NS}}$ & $0.051^{\mathrm{NS}}$ & $0.193^{\mathrm{NS}}$ & $0.447^{*}$ & $-0.819^{* *}$ & \\
\hline \multirow{2}{*}{ G Y } & $\mathrm{P}$ & $-0.141^{\mathrm{NS}}$ & $-0.359^{\circ}$ & $0.062^{\mathrm{NS}}$ & 0.354 & $-0.317^{\circ}$ & $0.064^{\mathrm{NS}}$ & $0.104^{\mathrm{NS}}$ & $0.424^{\prime \prime}$ & $0.330^{\circ}$ & $0.214^{\mathrm{NS}}$ & $0.181^{\mathrm{NS}}$ & $-0.085^{\mathrm{NS}}$ & $0.499^{*}$ & -0.474 \\
\hline & G & $-0.143^{\mathrm{NS}}$ & $-0.375^{\circ}$ & $0.063^{\mathrm{NS}}$ & $0.420^{* \prime}$ & $-0.340^{\circ}$ & $0.080^{\mathrm{NS}}$ & $0.224^{\mathrm{NS}}$ & $0.498^{\prime \prime}$ & $0.331^{\circ}$ & $0.224^{\mathrm{NS}}$ & $0.192^{\mathrm{NS}}$ & $-0.085^{\mathrm{NS}}$ & $0.501^{\prime \prime}$ & -0.475 \\
\hline
\end{tabular}

Where, ${ }^{* *}$ and significant at $1 \%$ and $5 \%$ level of significance, $N S=$ =non significant

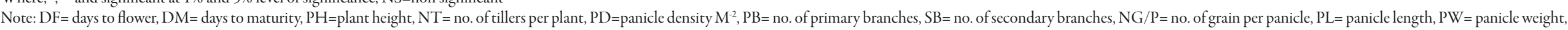
$\mathrm{GW}=100$ grain weight, $\mathrm{GL}=$ grain length, $\mathrm{GB}=$ grain breadth and $\mathrm{GY}=$ grain yield 
学 Tab. 4. Direct and indirect effects of fourteen agro-morphological characters on grain yield in red rice ecotypes from temperate region of Kashmir, India

\begin{tabular}{|c|c|c|c|c|c|c|c|c|c|c|c|c|c|c|c|}
\hline Characters & & $\mathrm{DF}$ & $\mathrm{DM}$ & $\mathrm{PH}$ & NT & $\mathrm{PD}$ & $\mathrm{PB}$ & SB & NG & $\mathrm{PL}$ & PW & GW & GL & GB & $\mathrm{L}: \mathrm{B}$ \\
\hline \multirow{2}{*}{ DF } & $\mathrm{P}$ & 0.126 & -0.098 & 0.007 & 0.022 & -0.034 & 0.010 & -0.000 & -0.392 & 0.261 & -0.012 & -0.066 & 0.236 & -0.596 & 0.396 \\
\hline & G & 5.514 & 0.433 & 0.511 & -1.521 & 1.002 & -0.101 & 0.006 & -5.137 & 0.174 & -0.299 & -0.003 & 0.554 & -2.722 & 1.447 \\
\hline \multirow{2}{*}{$\mathrm{DM}$} & $\mathrm{P}$ & 0.017 & -0.704 & 0.007 & 0.022 & 0.002 & -0.062 & 0.000 & -0.145 & 0.573 & -0.087 & -0.161 & 0.381 & -0.312 & 0.109 \\
\hline & G & 0.860 & 2.774 & 0.527 & -1.376 & -0.195 & 0.180 & -0.092 & -1.748 & 0.383 & -1.527 & -0.009 & 0.897 & -1.450 & 0.400 \\
\hline \multirow{2}{*}{$\mathrm{PH}$} & $\mathrm{P}$ & 0.031 & -0.181 & 0.029 & -0.056 & 0.147 & -0.238 & 0.000 & -0.232 & 0.557 & -0.068 & 0.076 & 0.074 & -0.229 & 0.152 \\
\hline & G & 1.435 & 0.744 & 1.965 & 3.927 & -4.483 & 0.577 & -0.105 & -2.871 & 0.357 & -1.188 & 0.004 & 0.175 & -1.015 & 0.539 \\
\hline \multirow{2}{*}{ NT } & $\mathrm{P}$ & 0.022 & -0.127 & -0.013 & 0.122 & -0.150 & 0.256 & 0.000 & 0.005 & -0.472 & 0.079 & -0.234 & 0.378 & -0.284 & 0.065 \\
\hline & G & 1.180 & 0.537 & -1.085 & -7.11 & 5.610 & -0.679 & -0.165 & 0.112 & -0.358 & 1.818 & -0.014 & 1.028 & -1.562 & 0.269 \\
\hline \multirow{2}{*}{ PD } & $\mathrm{P}$ & 0.020 & 0.007 & -0.019 & 0.085 & -0.367 & 0.239 & -0.000 & -0.011 & -0.497 & 0.116 & -0.156 & 0.248 & -0.314 & 0.182 \\
\hline & G & 0.884 & -0.086 & -1.410 & -6.385 & 6.248 & -0.638 & -0.038 & -0.287 & -0.330 & 1.908 & -0.010 & 0.603 & -1.464 & 0.666 \\
\hline \multirow{2}{*}{$\mathrm{PB}$} & $\mathrm{P}$ & -0.003 & -0.121 & 0.019 & -0.086 & 0.142 & -0.363 & -0.00 & -0.074 & 0.747 & -0.099 & 0.177 & -0.493 & 0.021 & 0.198 \\
\hline & G & -0.726 & 0.652 & 1.473 & 6.277 & -5.179 & 0.769 & 0.152 & -1.162 & 0.550 & -2.343 & 0.010 & -1.309 & 0.136 & 0.778 \\
\hline \multirow{2}{*}{ SB } & $\mathrm{P}$ & 0.007 & 0.025 & -0.001 & -0.015 & -0.016 & -0.039 & -0.900 & -0.000 & 0.451 & -0.068 & -0.040 & -0.248 & 0.056 & -0.004 \\
\hline & G & 0.038 & -0.279 & -0.226 & 1.284 & -0.265 & 0.127 & 0.918 & 0.905 & 0.690 & -2.115 & -0.014 & -1.478 & 0.656 & -0.017 \\
\hline \multirow{2}{*}{ NG } & $\mathrm{P}$ & 0.089 & -0.184 & 0.012 & -0.001 & -0.004 & -0.048 & 0.000 & -0.554 & 0.473 & 0.026 & 0.011 & 0.195 & -0.559 & 0.392 \\
\hline & G & 4.294 & 0.735 & 0.855 & 0.120 & 0.272 & 0.135 & -0.125 & -6.597 & 0.313 & 0.486 & 0.000 & 0.478 & -2.548 & 1.421 \\
\hline \multirow{2}{*}{ PL } & $\mathrm{P}$ & 0.024 & -0.300 & 0.012 & -0.043 & 0.079 & -0.202 & -0.000 & -0.195 & 1.345 & -0.150 & -0.039 & -0.215 & -0.098 & 0.113 \\
\hline & G & 1.116 & 1.237 & 0.816 & 2.968 & -2.399 & 0.492 & 0.736 & -2.409 & 0.859 & -2.553 & -0.002 & -0.502 & -0.431 & 0.401 \\
\hline \multirow{2}{*}{ PW } & $\mathrm{P}$ & 0.006 & -0.234 & 0.007 & -0.037 & 0.096 & -0.138 & -0.000 & 0.055 & 0.770 & -0.262 & -0.050 & -0.112 & 0.068 & 0.045 \\
\hline & G & 0.385 & 0.988 & 0.544 & 3.016 & -2.782 & 0.420 & 0.453 & 0.7491 & 0.512 & -4.286 & -0.003 & -0.288 & 0.329 & 0.185 \\
\hline \multirow{2}{*}{ GW } & P & -0.017 & 0.238 & 0.004 & -0.060 & 0.070 & -0.135 & 0.000 & -0.013 & -0.112 & 0.027 & 0.477 & -0.722 & 0.240 & 0.181 \\
\hline & G & -0.841 & -1.020 & 0.338 & 4.076 & -2.587 & 0.336 & -0.521 & -0.186 & -0.077 & 0.550 & 0.024 & -1.775 & 1.177 & 0.696 \\
\hline \multirow{2}{*}{ GL } & $\mathrm{P}$ & -0.023 & 0.214 & -0.001 & -0.037 & 0.042 & -0.143 & -0.000 & 0.086 & 0.231 & -0.023 & 0.275 & -1.253 & 0.089 & 0.457 \\
\hline & G & -1.044 & -0.851 & -0.117 & 2.499 & -1.287 & 0.344 & 0.463 & 1.079 & 0.147 & -0.422 & 0.014 & -2.926 & 0.401 & 1.614 \\
\hline \multirow{2}{*}{ GB } & $\mathrm{P}$ & -0.074 & 0.217 & -0.006 & -0.034 & 0.067 & -0.007 & -0.000 & 0.306 & -0.130 & -0.017 & 0.113 & -0.11 & 1.012 & -0.835 \\
\hline & G & -3.364 & -0.901 & -0.447 & 2.490 & -2.050 & 0.023 & 0.135 & 3.768 & -0.083 & -0.316 & 0.006 & -0.263 & 4.461 & -2.957 \\
\hline \multirow{3}{*}{$\mathrm{L}: \mathrm{B}$} & $\mathrm{P}$ & 0.048 & -0.074 & 0.004 & 0.007 & -0.038 & -0.070 & 0.000 & -0.212 & 0.149 & -0.011 & 0.084 & -0.559 & -0.826 & 1.024 \\
\hline & G & 2.211 & 0.307 & 0.293 & -0.531 & 1.154 & 0.165 & -0.004 & -2.597 & 0.095 & -0.220 & 0.004 & -1.308 & -3.655 & 3.610 \\
\hline & sidual & & & & & & & & 0.075 & & & & & & \\
\hline
\end{tabular}

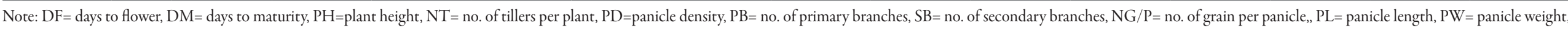
$\mathrm{GW}=100$ grain weight, $\mathrm{GL}=$ grain length, $\mathrm{GB}=$ grain breadth, $\mathrm{L}: \mathrm{B}=$ length breadth ratio and $\mathrm{GY}=$ grain yield 
406

coefficient analysis are appended in Tab. 4 revealed that day to flowering and panicle density exhibited high positive direct effect and significant positive association with grain yield followed by days to maturity and plant height at genotypic level. Results on importance of direct effect of number grain per panicles were also reported by several researchers (Siva Kumar and Bapu, 2005; Panwar and Ali, 2007; Panwar, 2006). This indicates that, if other factors are held constant, an increase in days to 50 per cent flowering individually will reflect in an increased yield. The residual effect $(0.075)$ was very low, indicating that much of the variation in yield has been accounted by the characters studied and that the choice of characters was appropriate. The overall results indicated that there is adequate genetic variability present in the material studied. The broad sense heritability, genetic advance and correlation analysis of the study revealed that the number of panicles per plant, panicle weight and the number of grains per panicle were the most important yield components. These characters also showed moderate to high heritability and genetic advance as percentage of mean. Therefore, the results suggest that the number of panicles per plant, panicle length and the number of grains per panicle are important yield contributing traits and selection based on these traits would be most effective. It could be concluded that more emphasis should be given on days to $50 \%$ flowering, panicle length, panicle density, number of productive tiller per plant to bring simultaneous improvement of yield and it's attributes in local rice genotypes as they showed high correlation in addition to maximum direct effects on yield.

\section{References}

Babu SG, Lavanya GR, Singh AP (2011). Genetic variability for grain yield and character association studies in upland rice (Oryza sativa L.). Environ Ecol 29(1):164-168.

Bhandarkar S, Ravindra V, Kumar A (2002). Genetic variability and correlation analysis in early duration rice. Plant Arch 2(1):95-98.

Bhardwaj C, Mishra R, Satyavathi CT, Rao SK, Kumar KS (2007). Genetic variability, heritability and genetic advance in some new plant type based crosses of rice (Oryza sativa L.). Indian J Agric Res 41 (3):189- 194.

Bhattacharjee P, Singhal RS, Kulkarni PR (2002). Basmati rice: A review. Int J Food Sci and Tech 37(1):1-12.

Chaudhary RC (2003). Specialty rices of the world: Effect of WTO and IPR on its production trend and marketing. Journal of Food, Agriculture and Environment 1(2):34-41.

Dewey DR, Lu KH (1959). A correlation and path coefficient analysis of components of crested wheat grass seed production. Agron J 51(9):515-518.

Eradasappa E, Nadarajan N, Ganapathy KN, Shanthala J, Satish RG (2007). Correlation and path analysis for yield and its attributing traits in rice (Oryza sativa L.). Crop Res 34:156159.
Idris AE, Justin FJ, Dagash YMI, Abuali AI (2012). Genetic variability and inter relationship between yield and yield components in some rice genotypes. American Journal of Experimental Agriculture 2(2):233-239.

Iftekharuddeula M, Hassan S, Islam MJ, Badshah MA, Islam MR, Khaleda A (2001). Genetic evaluation and selection criteria of Hybrid rice in irrigated ecosystem of Bangladesh. Pak J Bio Sci 4(7):790-791.

Itani T, Ogawa M (2004). History and recent trends of red rice in Japan. Japanese Journal of Crop Science 73(2):137-147.

Jayasudha S, Sharma D (2010). Genetic parameters of variability, correlation and path coefficient for grain yield and physiological traits in rice (Oryza sativa L.) under shallow lowland situation. Electronic J Plant Breed 1(5):33-38.

Johnson HW, Robinson HF, Comstock RE (1955). Estimates of genetic and environmental variability in soybean. Agronomy J 47(7):314-318.

Kuldeep T, Bathshwar K, Ramesh B, Tomer A (2004). Genetic variability and correlations for some seedlings and mature plant traits in 70 genotypes of rice. Res Crops 5(1):60-65.

Oka HI (1988). Origin of cultivated rice, Japan Scientific Society Press, Tokyo, $254 \mathrm{p}$.

Panwar LL (2006). Character association and path analysis in rice (Oryza sativa L.). Ann Agrl Res 27(3):257-260.

Panwar LL, Ali M (2007). Correlation and path analysis of yield and yield components in transplanted rice. Oryza 44(2):115-120.

Prajapati M, Singh CM, Suresh BG, Lavanya GR, Jadhav P (2011). Genetic parameters for grain yield and its component characters in rice. Electronic J Pl Breed 2(2):235-238.

Rood MA (2000). Red menace. Rice Journal 103:18-20.

Sabesan T, Suresh R, Saravanan K (2009). Genetic variability and correlation for yield and grain quality characters of rice grown in coastal saline lowland of Tamiluadu. Electronic J Plant Breed 1:56-59.

Sanghera GS, Kashyap SC (2012). Genetic parameters and selection indices in $\mathrm{F}_{3}$ progenies of hill rice genotypes. Not $\mathrm{Sci}$ Biol 4(4):110-114.

Sinha SK, Tripathi AK, Bisen UK (2004). Study of genetic variability and correlation coefficient analysis in midland landraces of rice. Annal of Agric Res 25(1):1-3.

Siva Kumar P, Kannan Bapu JR (2005). Character association in inter sub-specific rice hybrids involving wide compatible gene. Crop Res 30(2):208-210.

Yadav RK (2000). Studies on genetic variability for some quantitative Characters in rice (Oryza sativa $\mathrm{L}$ ). Advances in $\mathrm{Ag}$ ric Res 13:205-207.

Zhang M.W, Guo B-J, Chi J-W, Wei Z-C, Xu Z-H, Zhang Y, Zhang R-F (2005). Antioxidations and their correlation with total flavonoid and anthocyanin contents in different black rice varieties. Scientia Agricultura Sinica 38(7):13241331. 